\title{
Progression and reversibility of gliosis due to hydrocephalus in the H-Tx rat
}

\author{
Alexander G Shanku*1,2, Janet M Miller1,2 and James P McAllister ${ }^{1,2}$
}

\author{
Address: 'Departments of Neurosurgery and Physiology; Wayne State University School of Medicine and ${ }^{2}$ Department of Pediatric Neurosurgery, \\ Children's Hospital of Michigan. Detroit, Michigan 48201 \\ Email: Alexander G Shanku* - ashanku@med.wayne.edu \\ * Corresponding author
}

from 49th Annual Meeting of the Society for Research into Hydrocephalus and Spina Bifida

Barcelona, Spain, 29 June - 2 July 2005

Published: 30 December 2005

Cerebrospinal Fluid Research 2005, 2(Suppl I):S2 doi:10.1 I86/1743-8454-2-SI-S2

\section{Background}

Persistent gliosis, if present in shunt-dependent hydrocephalus, has the potential to alter the biomechanical properties of the brain, impair cerebral perfusion, and impede neuronal regeneration and plasticity. Determining the onset, progression, and reversibility of gliosis due to hydrocephalus is important in designing and implementing better clinical treatments for this disorder. Thus, gliosis was studied as the severity of hydrocephalus increased in five, twelve and twenty one day old untreated $\mathrm{H}$-Tx rats with congenital hydrocephalus and control littermates. Previous clinical and experimental studies have suggested that gliosis may persist after shunting, but these findings have been based on relatively short post-shunt survival times.

\section{Materials and methods}

In an effort to determine the effects of post-shunt recovery time on hydrocephalus-induced gliosis, we placed neonatal low-pressure ventriculo-subcutaneous shunts in 15 day old H-Tx rats with severe hydrocephalus, and sacrificed these animals 1 or 3 weeks later. Tissue from different regions of the cerebral cortex was processed for routine immunohistochemistry and Western blot procedures, and probed for both microglial (Isolectin B4, ILB4) and astrocytic (Glial Fibrillary Acidic Protein, GFAP) proteins.

\section{Results}

Western blot analysis revealed a marked increase in GFAP protein in both the occipital and parietal cortices of 12 day old hydrocephalic animals, suggesting that an increase in pressure was the trigger for the onset of gliosis. The dramatic rise in GFAP expression continued to be seen in both 21- and 36-day old untreated hydrocephalic animals as well. Shunting these hydrocephalic animals in order to reduce the increased intracranial pressure had a significant effect; both short and long recovery periods were able to reduce the amount of gliosis present, indicating that shunting is an effective treatment for reversing persistent gliosis. Immunohistochemical examination of GFAP followed similar up-regulation trends as seen in the Western blot analysis.

ILB4 blots indicated that in young hydrocephalic animals (5 \& 12 day animals) there was a trend toward up-regulation of microglia in response to hydrocephalus, but from day 21 and beyond, microglia levels decreased in response to injury. Shunted hydrocephalic animals exhibited microglia levels similar to those of control animals. On histochemical examinations, microglia appeared to be developmentally delayed in young hydrocephalic animals, and in the older 21 and 36 day hydrocephalic animals, they exhibited an activated morphology. Morphologically, microglia in shunted animals appear to be in a state of recovery; i.e. they are not as activated as in hydrocephalic animals, but still show some signs of reactivity.

\section{Conclusion}

Overall, these results suggest that reactive astrocytosis and microgliosis are associated with progressive untreated ventriculomegaly, but that appropriately timed shunting can reverse most of these effects. It remains to be seen, however, if gliosis behaves the same with persistent shunt malfunctions. 